\title{
Insights on Romantic Medieval Literature
}

\section{காதல் சிற்றிலக்கியங்களும் உள்ளடக்கங்களும்}

\section{R. Veerapathiran a, ${ }^{*}$}

a Department of Tamil, Karpagam Academy of Higher Education, Coimbatore-641021, Tamil Nadu, India.

*Corressponding author Cell: +91 9442365680; Email: veerapathiran.r@kahedu.edu.in

DOI: https://doi.org/10.34256/ijmrd2014

Received: 24-11-2020; Accepted: 14-12-2020; Published: 17-12-2020

Abstract: Literature (Tholkapiyar) originated under the name of love, as later epistemological grammars expanded on the levels of theft and chastity found in the Tholkapiyar period. In Sangam literature, the human love was sung over the leader and gradually became the divine love in the devotional literature and later turned into human love again in the cynical period. Romantic Medieval Literature were created in defiance of the notion that the leader should not be named in the songs, and that the hunting action was based on the side. It was a combination of love and heroism. Although Romantic Medieval Literature have declined due to the inability of later poets to combine love and heroism, romantic medieval literature has become an excellent literature in terms of subject matter and content analysis.

Keywords: Tholkapiyar, Tamil Literature, Romantic Medieval Literature, Sangam literature, Devotional literature.

\section{முன்னுரை}

பழந்தமிழ் இலக்கியங்களில் ுறப்பாடலைவிட அகப்பாடல்களே மிகுதியாக பாடப்பட்டுள்ளன. ஆணும் பெண்ணும் ஒருவர்மீது ஒருவர் செலுத்துகின்ற அன்பு அகம் என்றும் இவை தவிர்த்து அமைகின்ற அனைத்தும் புறம் என்றும் பழந்தமிழர் வரையறுத்துள்ளனர். அகம் எனினும் காதல் எனினும் பொருள் நோக்கில் ஒத்தனவே. எனினும் சங்க இலக்கியங்கள், பக்தி இலக்கியங்கள், காப்பியங்கள் போன்றவற்றில் ஆண்-பெண் உறவு நிலையான காதலை அகம் என்றே பதிவு செய்துள்ளன. இடைக்காலத்தில்தான் இந்நிலைமாறி காதல் என்னும் பெயரிலேயே சிற்றிலக்கியங்கள் படைக்கப்பட்டன. சிற்றிலக்கியங்களுக்கு இலக்கணம் கூறும் பாட்டியல் நூல்கள் குறிப்பிட்டுள்ள 96 வகை இலக்கியங்களில் காதல் என்னும் சிற்றிலக்கியம் இடம்பெறவில்லை (Ponnusamy, 2004). எனினும் இக்காதல் இலக்கியம் சங்க இலக்கியப் பாடல்கள் போன்று கருத்தமைவில் மக்களிடம் செல்வாக்குப் பெற்று விளங்குவதை அதன் உள்ளடக்கப் பாகுபாட்டினைக் கொண்டு, இலக்கிய அமைப்பு முறைகளை ஆய்ந்து அறிவதாக இக்கட்டுரை அமைகின்றது.

\section{காதல் இலக்கியம் பொருள் விளக்கம்}

“காதல் என்ற சொல்லிற்கு அன்பு, காமவிச்சை, பக்தி, வேட்கை, ஆவல், பிரபந்த விசேடம், கொல்லுகை, தாக்குகை, தறிக்கை, ஆந்தை முதலியவற்றின் ஒலி போன்ற பொருள்களைத் தருகிறது சென்னைப் பல்கலைக்கழகப் பேரகராதி" தொல்காப்பியத்திலேயே இச்சொல்லின் ஆட்சி காணப்படுகின்றது. விருப்பம் என்ற அடிப்படைப் பொருண்மை, அன்பு ஆகியன மிகுதியாகப் பயின்றுவருகின்றன. அக உணர்வான காதல் அகப்பொருள் எனக் குறிக்கப்படும் பெரும்பான்மை நிலையை பழங்காலத்தில் காண்கிறோம். அகப்பொருளிலும் களவே காதலோடு ஒ ஒத்திணைந்து அமைகின்றது. 
தொல்காப்பியம் ஐந்திணைப் பகுப்புக் கூறினும் களவு, கற்பு எனும் இருவகை நிலைக்கே முதலிடம் அளிக்கின்றது. சங்க இலக்கியத் தொகுப்புகள் திணைக்கு முதலிடம் அளித்ததைத் தெளிவாக உணர முடிகின்றது. ஐந்திணையில் குறிஞ்சி, களவுக் காதலை மிகுதியாகப் பேசியுள்ளது (Tholkapiyam - Porulathigaram, 1969). பிற்காலக் கோவையிலக்கியம் களவுக்குப் பேரிடம் அளித்து கற்பில் முடிகின்றது. சங்கக் குறிஞ்சிப்பாட்டு போன்ற நெடும்பாடல்கள் அறத்தொடு நிற்றல் வாயிலாகத் தலைவியின் களவுக் காதலை வெளிப்படுத்தியது. இவ்வாறு களவுக் காதலை வெளிப்படுத்தும் தனிப்பாடல்களே சங்க இலக்கியங்களில் பெரும்பான்மையாகக் காணப்படுகின்றன. பின்னர் தலைவன்தலைவி இணைவை விரித்து அகப்பொருளை உள்ளிறுத்தி இலக்கியங்கள் படைக்கப்பட்டன. இவ்வாறு அமையும் இலக்கியங்களைக் காதல் இலக்கியங்கள் என்று கொள்ளல் தகும்.

\section{சங்க இலக்கியத்தில் காதல்}

கடைச்சங்கத்தில் தோன்றிய எட்டுத்தொகையும் பத்துப்பாட்டும் தமிழ் இலக்கியங்களில் மமத்த இலக்கியங்கள், முதன்மை இலக்கியங்கள் என்ற பெருமையைப் பெறுகின்றன. இவ்விலக்கியங்கள் அகம், புறம் என்ற இரண்டு நிலைகளிலும் அக்காலத்து மக்களின் வாழ்வியலை உணர்த்துவதாய் அமைகின்றன. களவு, கற்பு என்ற இரண்டு நிலைகளிலே இந்த அகப்பாடுபொருள் கையாளப்பட்டிருக்கிறது. முன்பின் அறியாத தலைவனும் தலைவியும் காதல் கொள்ளும் நிலையை,

“ஒன்றே வேறே என்றிரு பால்வயின்

ஒன்றி உயர்ந்த பால தாணையின்

ஒத்த கிழவனும் கிழத்தியும் காண்ப" (தொல்காப்பியம், நூற்பா - 1039)

என்ற நூற்பாவின் வழி தொல்காப்பியர் விளக்குகிறார். இதனைச் சங்க இலக்கியக் குறுந்தொகைப் பாடல்,

"யாயும் ஞாயும் யாரா கியரோ

எந்தையும் நுந்தையும் எம்முறை கேளிர்

யானும் நீயும் எவ்வழி யறிதும்

செம்புலப் பெயல்நீர் போல

அன்புடை நெஞ்சம் தாங்கலந்தனவே" (குறுந்தொகை, பாடல் - 40)

என்று தலைவன் - தலைவியின் அகவாழ்க்கையை விளக்குகிறது.

தலைவனும் தலைவியும் காதல் வாழ்வில் நுகரும் இன்பத்தை உலகிற்குத் தெரியப்படுத்த முடியாது என்பதால்தான் இன்னாரென்று கூறாமல்

"மக்கள் நுதலிய அகணைந்திணையும்

சுட்டி ஒருவர் பெயர்கொளப் பெறாஅர்" (தொல்காப்பியம், நூற்பா - 1000)

என்று தொல்காப்பியர் காதலுக்கு ஒரு வரையறை வைத்தார்.

\section{இடைக்காலத்தில் காதல்}

சங்க காலத்தில் ஒருவனும் ஒருத்தியும் கொண்டிருந்த ஓருயிர்ப் புணர்வாகிய காதல் இடைக்காலத்தில் கடவுட் காதலாக மாறியது. காதலால் இறைவனை அடையத் துடிக்கும் ஆன்மா தன்னைப் பெண்ணாகப் பாவித்து அன்பு செலுத்துகின்றது. சங்க இலக்கியங்களில் போற்றப்பெறும் காதல் ஆண் பெண் உடல் பற்றியதும் உயிர்ப் பற்றியதும் ஆகும். ஆனால் பக்தி இலக்கியங்களில் காணப்பெறும் காதலோ முழுக்கமுழுக்க உயிர்ப் பற்றியதே ஆகும். 
தொல்காப்பியர் குறிப்பிட்ட “சுட்டி ஒருவர் பெயர்கொளப் பெறாஅர்" என்ற நிலை உடைந்து அக இலக்கியத்தில் தலைவன் தலைவியரின் பெயரும் குறிப்பிடப்படலாம் என்ற நிலை இடைக்காலத்தில் ஏற்பட்டது. இதன் விளைவாக இடைக்கால இலக்கியங்களான காப்பிய இலக்கியம், பக்தி இலக்கியம், சிற்றிலக்கியம் போன்ற இலக்கியங்களில் அகம் (காதல்) பற்றிய செய்திகளை விளக்கும் பாடல்களில் தலைவன் தலைவியரின் பெயர் சுட்டப்பெற்றன.

பக்தி இலக்கிய வரலாற்றில் ஆண்டாள் இறைவனிடத்தில் காதல் கொண்டு காதல் சுவை சொட்டச் சொட்டச் சொட்டப் பாடியதும், மாணிக்கவாசகர் தன்னைப் பெண்ணாகப் பாவித்து சிவபெருமானிடத்திலும், நம்மாழ்வார் திருமாலிடத்திலும், வள்ளலார் இங்கிதமாலை என்னும் நூலில் நாயக-நாயகி பாவனையில் பாடியிருக்கும் செய்திகள் நமக்குக் கிடைக்கின்றன.

\section{காதல் இலக்கியங்கள்}

பக்தி இலக்கியக் காலத்தில் இறைவன்மீது காதல் கொண்டு பாடிய நிலைமாறி, சிற்றிலக்கியக் காலத்தில் மீண்டும் மனிதர்கள் மீதும் காதல் இலக்கியம் பாடும் நிலை ஏற்பட்டது. காதல் செய்திகளை முதன்மைப்படுத்தி பல்வேறு சிற்றிலக்கியங்கள் இக்கால கட்டத்தில் தோன்றின. உலா, தூது போன்ற இலக்கியங்கள் பெயரில் வேறுபாடு காணப்படினும் அவ்விலக்கியங்களில் காதல் சுவைகள் பெரிதும் காணப்படுகின்றன.

காதலைப் பொருண்மையாக வைத்து பல சிற்றிலக்கியங்கள் படைக்கப்பட்ட நிலையில் “காதல்" என்ற பெயரிலேயே இலக்கியங்கள் தோன்ற ஆரம்பித்தன. மேற்குறிப்பிட்ட காதல்கள் எல்லாம் பெரும்பான்மையும் இயல்பாய் எழுபவை. புதிதாக எதையும் எதிர்நோக்கிப் படைக்கப்படாதவை. ஆனால், இங்கு நாம் காணும் காதல் அதனினின்று மாறுபட்டது. பல்வேறு அரசியல், சூழ்நிலைகளின் காரணமாக வறுமையில் வாடிய புலவன் தலைவனைப் பாடிப் பரிசில் பெறும் நோக்கத்திற்காகக் காதல் சிற்றிலக்கியங்கள் படைக்கப்பட்டன.

\section{காதல் இலக்கியத்தின் இலக்கணம்}

காதல் சிற்றிலக்கியத்திற்குப் பெரும்பாலான பாட்டியல் நூல்கள் இலக்கணம் கூறவில்லை. பாட்டியல் கூறாத சிற்றிலக்கியம் எனப் பகுத்துச் செல்லும் அறிஞர்கள் இக்காதலைப் "பாட்டியல் கூறா" வகையில் அடக்குகின்றனர். காதல் இலக்கியப் பதிப்பாளர்கள் சிலரும் காதல் இலக்கியத்திற்குப் பாட்டியல் நூல்கள் இலக்கணம் ஆக்கவில்லை என்கின்றனர்.

பாட்டியல் நூல்களில் சுவாமிநாதம்,

“கொண்டமயல் ஈரடிக்கண்ணியில் இசை காதலுக்கோ" (சுவாமிநாதம், நூற்பா - 17)

என்று காதல் இலக்கியத்திற்கு இலக்கணம் சுட்டுகின்றது. இவ்விலக்கணமும் காதல் இலக்கியத்தின் முழு இலக்கணமாக அமையவில்லை. காதல் இலக்கியம் இரண்டடிக் கண்ணியாகப் பாடப்படுவது என்பது மட்டும் இதன் மூலம் அறிய முடிகின்றது.

காதல் சிற்றிலக்கியங்களில் தலைவி பற்றிய வருணனை இடம்பெறுகின்றது. அது கேசாதிபாதமாக அமைகின்றது. பிரபந்தத்திரட்டு இக்காதலை பாதாதிகேசத்திற்குப் புறனடையாய்க் காட்டுகின்றது.

“இன்னதுக்குப் புறனடையாய் காதலென்று பெயரா

யிசைத்தார்கள் பெரியோர்கள்" (பிரபந்தத்திரட்டு, நூற்பா - 476)

என்று மட்டும் கூறுகிறது. இவற்றால் ஈரடிக் கண்ணியில் பாடப்படுவது என்று மட்டும் அறிய முடிகிறதேயன்றி வரையறை ஏதும் பெறமுடியவில்லை. கண்ணிகள் இத்தனைதான் அமைய வேண்டும் என்ற வரையறையும் அமையவில்லை.

காதல் இலக்கியத்தின் அமைப்புமுறை 
பாட்டுடைத் தலைவன் மலை, ஆறு முதலிய பத்து உறுப்புகளையும் பிற சிறப்புகளையும் கூறித் தொடங்கும். பாட்டுடைத் தலைவன் ஒருநாள் வேட்டைக்குச் செல்கிறான். அவனைக் கண்ட பெண்கள் வேட்கையுற்றுப் புலம்புகிறார்கள். வீரர்கள் வஞ்சினம் கூறிக்கொண்டு விரைந்து செல்கிறார்கள். கானவர்களும் அவர்களுடன் வேட்டைக் கருவிகளுடன் இணைந்து கொள்கின்றனர்.

வேட்டையின்போது அரசன் ஒரு மானைக் கண்டு, அதனைப் பின் தொடர்ந்து செல்கிறான். தோழியருடன் மலர்க் கொய்ய வந்த தலைவி தனியே அப்பக்கமாக வருகிறாள். மானைத் தொடர்ந்து சென்ற தலைவன் அப்பெண்ணைக் காண்கிறான். இருவரும் கூடிக் கலந்து இன்புறுகின்றனர். தலைவனைக் காணாது வீரர்கள் அவனைத் தேடி வர தலைவனும் தலைவியும் பிரிந்துவிடுகின்றனர். பிரிவை எண்ணிய தலைவி வருத்தமுறுகிறாள். அவள் துயரைக்கண்ட தோழியர் அவளை வட்டிற்கு அழைத்துச் செல்கின்றனர். செய்தியறிந்த தாய் புலம்புகிறாள். தலைவனையும் ஏசுகிறாள்.

தலைவன், அவளை அழைத்துவரப் பல்லக்கு அனுப்புகிறான். அதனை அறிந்த தாய் மகிழ்ச்சியுடன் வாழ்த்தித் தலைவியை அனுப்புகிறாள். இருவரும் இன்புற்று வாழ்ந்து புதல்வரைப் பெற்றுச் சிறக்கின்றனர்.

பெரும்பாலான காதல் சிற்றிலக்கியங்கள் இம்முறையில் அமைகின்றன. சில இலக்கியங்கள் மாற்றம் பெறுகின்றன. சில படைப்பாளர்கள் தங்களின் எண்ணத்தின் மீது கொண்ட காதலைப் பாடுவதையும் இவ்விலக்கிய அமைப்புக்குள் அடக்க முயல்கின்றனர்.

காதலைத் தனித்துப்பாடும் இந்நிலை சங்க இலக்கியங்களிலேயே இடம் பெற்றுள்ளதைக் காணமுடிகின்றது. இவ்வகையில் அமையும் காதல் இலக்கியங்களில் தனிமனிதனே மிகுதியும் பாட்டுடைத் தலைவனாக இடம்பெறுகிறான்.

\section{காதல் இலக்கிய பெயரமைப்பு}

இக்காதல் இலக்கியம் பாடுபொருளால் பெயர்பெறும் சிற்றிலக்கிய வகையாகும். இச்சிற்றிலக்கிய பெயரமைப்பு முறையில் மூன்று நிலைகளைக் காணமுடிகின்றது. பாட்டுடைத் தலைவன் பெயருடன் காதல் என்று அமைவது ஒன்று. பாட்டுடைத் தலைவன் பெயருடன் பேரில் காதல் என்று அமைவது மற்றொன்று. பாட்டுடைத் தலைவன் பெயருடன் மீது காதல் என்று அமைவது வேறொன்று. இஃதல்லாமல் சிலவற்றில் பிரபந்தம் என இணைத்தும் பெயரமைவது உண்டு. இவை முறையே கூளப்பநாயக்கன் காதல், கருப்பண்ணசாமி பேரில் காதல், சர்க்கரை மன்றாடியார்மீது காதல், சண்முகஞ் செட்டியார் காதல் பிரபந்தம் என அமைகின்றன. இதில் பேரில் காதல் என்பது மட்டும் இறைவனைப் பாட்டுடைத் தலைவனாகக் கொண்ட காதல் இலக்கியங்களில் காணப்படுகிறது.

\section{பாடுபொருள்}

ஓவ்வொரு இ இலக்கியமும் ஏதேனும் ஒ ஒரு பாடுபொருளை மையமாகக் கொண்டு பாடப்படுகின்றது. அப்பாடுபொருள் அகமாகவும் இருக்கலாம் புறமாகவும் இருக்கலாம். அகம் புறம் என்ற இரு நிலைகளையும் கலந்தும் அமைபவையாகவும் இருக்கலாம்.

சங்க காலத்தில் காதலும் வீரமும் முதன்மையாகக் கருதப்பட்டது. சங்க காலத்திற்குப் பின் காதலையும் வீரத்தையும் இணைத்து இயற்றிய இலக்கியம் இருப்பதாகத் தெரியவில்லை. முத்தொள்ளாயிரம் ஓரளவுக்கு இதை நிறைவு செய்துள்ளது. காதல் சிற்றிலக்கியத்தில் காதல் முதன்மைப் பாடுபொருளாக அமைந்துள்ளது. வீரத்தைப் புலப்படுத்த வேட்டையாடுதல் குறிப்பிடப்பட்டுள்ளது. ஆகவே காதல் சிற்றிலக்கியங்கள் காதலும் வீரமும் இணைந்தது என்றே கூறலாம்.

காதல் சிற்றிலக்கியங்கள் அனைத்தும் ஒரே மாதிரியான பாடுபொருளைக் கொண்டு அமைந்துள்ளன என்று கூறமுடியாது. சில இலக்கியங்கள் பெயரளவிலேயே காதல் என அமைந்து அதன் பாடுபொருளும், முறையும், நிகழ்ச்சிகளும் வேறுபடுகின்றன. அகத்தியர் பள்ளு, பள்ளு இலக்கியத்தின் பாடுபொருளில் இருந்து முற்றிலும் மாறுபட்டு மருத்துவ முறைகளை முதன்மையாகக் 
கொண்டு இயற்றப்பட்டுள்ளது. இதேபோல சில காதல் சிற்றிலக்கியங்களும் பாடுபொருளில் வேறுபட்டு காணப்படுகின்றன.

மனுநீதிக்காதல் இலக்கியம் மனுநீதிகளின் சிறப்பைப் பற்றிப் பேசுகின்றது. அனைத்து வகையினருக்கும் ஒழுக்கங்களையும், நீதிகளையும் எடுத்துரைக்கின்றது. இந்நூலின் அவையடக்கத்தில் திருவள்ளுவர், ஒளவையார் போன்றோரின் ஒழுக்க நெறிகளைப் பின்பற்றி நீதிகளை உரைக்கின்றதேயன்றி, இதற்கும் காதல் இலக்கியங்களுக்கும் தொடர்பில்லை என்பதை,

"மாலைசதக மனுநீதி சாரமென்று

ஞாலமதிற் கவிஞர் நவின்றார் பலநூலால் இந்நூலைக்

காதலுடன் யானுமொர் காதலெனப்பா டிவைத்தேன்" (மனுநீதிக்காதல்,கண்ணி -1)

என்ற பாடலடிகளால் இந்நூலின் ஆசிரியர் தெய்வச்சிலையா பிள்ளையவர்கள் குறிப்பிடுவதை அறியலாம். இக்காதல் தெய்வச்சிலையாபிள்ளை மனுநீதிகளின் மீது கொண்டிருந்த காதலையே நமக்குக் காட்டுகின்றது.

கந்தர் காதல் தலைவிக்குத் தோழி அறத்தொடு நிற்றல் துறையாக அமைந்துள்ளது. கதிர்காமக் காதல் முருகனின் அருமை பெருமைகளை அடியார்க்கருளும் திறத்தை எடுத்தோதும் நிலையில் அமைந்துள்ளது. மேற்குறிப்பிட்ட காதல் இலக்கியங்கள் பெயரளவில் காதல் என அமைந்துள்ளதே தவிர காதல் இலக்கியப் பாங்கினைப் பெறவில்லை (Subramaniyan, 1984; Kodumanal Literatures, 1981).

\section{காதலும் வீரமும்}

ஒத்த அன்புடைய தலைவனும் தலைவியும் தாமே எதிர்பட்டு காதல் கொள்ளுதல் என்பதையே அக இலக்கியங்கள் சுட்டுகின்றன. இக்காதல் இலக்கியத்தில் தலைவனையும் தலைவியையும் சந்திக்க வைப்பதற்காக வேட்டையாடுதல் என்னும் வீரச்செயல் குறிப்பிடப்படுகின்றது. தலைவன் வேட்டையாடும் போது அவனிடமிருந்து தப்பியோடிய விலங்கினைத் துரத்திச் செல்லும்போது ஆங்குத் தலைவியைக் காண்பதாகப் புனையப்பட்டுள்ளது.

தலைவன் தலைவியைச் சந்திப்பதற்கு எல்லா காதல் இலக்கியங்களும் மான்வேட்டையாடுதலைப் பயன்படுத்தியுள்ளன. கந்தசாமிக் காதல், பொன்னையன் காதல் இவ்விரு இலக்கியங்கள் மட்டும் புலி வேட்டையைப் பயன்படுத்தியுள்ளன. வேட்டைக்காகத் தொடரும் போதுதான் எல்லாத் தலைவர்களும் தலைவியைக் காண்கின்றனர்.

"பதுங்கி யிருந்தொரு பதினா றடிவேங்கை

குதிங்கால் நரம்பலுங்கக் கொக்கரித்துச் சத்தமிட்டு

போரபுலி யைத்துடர்ந்து பூங்கா வனத்தருகே" (பொன்னையன் காதல், கண்ணி - 98)

என்று தாவிக்குதித்தோடிய புலியைப் பின் தொடர்ந்து தலைவன் ஓடியதாகக் கந்தசாமிக் காதல் கண்ணிகளால் அறியமுடிகின்றது. மான் வேட்டையைவிட புலிவேட்டையே வீரத்திற்குப் பெருமை என்ற எண்ணத்தில் ஆசிரியர் இவ்வாறு பயன்படுத்தி இருக்கலாம் என்று எண்ணத்தோன்றுகிறது.

\section{தலைவியின் கனவு}

கனவு காணுதல் என்பது ஒவ்வொருவருக்கும் இயல்பாய் அனிச்சை செயலாக நடக்கக் கூடியது. நமது எண்ணங்களும் உள்மனதின் ஆசைகளும் கனவுகள் நிறைவேற்றுகின்றன. சில சமயம் கனவில் கண்ட நிகழ்வுகளும் நனவில் நிகழ்கின்றன. இலக்கியங்களில் காணப்படும் கனவு நிலைகள் பெரும்பாலும் நனவாக மாறியதையே காட்டுகின்றன. 
சங்க இலக்கியத்தில் கூறப்படும் கனவுக் காட்சிகள் தலைவனைப் பிரிந்த தலைவி, தலைவனோடு இருக்கும்போது நுகர்ந்த இன்ப உணர்வுகளை, தலைவி கனவு மூலம் மீண்டும் அந்த ஏக்கத்தால் நுகர்வதாகக் கூறப்பட்டுள்ளன. இக்காதல் இலக்கியத்தில் முற்றிலும் வேறுபட்டு காதல் வயப்பட்டு தலைவி ஓர் ஆடவனுடன் கலந்திருப்பதாகச் சொல்லப்பட்டுள்ளது.

கந்தசாமிக் காதல் தலைவியான முத்துமாலை பூங்காவில் மலர் பறிக்கும்போது முருகக்கடவுள் வந்து அவளுடன் கலந்ததாகக் கனவு கண்டதை தோழியிடம் கூறுகிறாள். இந்நிலையை,

"பூவெடுத்து கொண்டந்தப் பூங்காவில் நானிருக்கத்

தேவேந் திரன்புதல்வி தெவியானை பங்கன்வந்து

என்னைக் கலந்திருக்க எந்தனிட சொப்பனத்தில்

தன்மையாய்க் கண்டனென்று தாதியுடன் தானுத்தாள்"

(கந்தசாமிக்காதல், கண்ணி - 231)

என்று இங்குப் போற்றப்படும் காதல் உணர்வு மரபுகளை மீறிய காதல் உணர்வாகச் சுட்டப்படுகிறது .

\section{மனைவி பெயர் சுட்டல்}

பெரும்பாலும் காதல் சிற்றிலக்கியங்களில் தலைவனுக்கு மனைவி கூறப்படுவதில்லை. தலைவன் தலைவியைக் கண்டு காதல் கொள்வது, இணைவது போன்ற செய்திகள்தான் அனைத்து வகை இலக்கியங்களிலும் கூறப்பட்டுள்ளன. ஆனால் காதல் சிற்றிலக்கியத்தில் கந்தசாமிக்காதல் இலக்கியத்தில் மட்டுமே தலைவனுக்கு மனைவி இருப்பதாகக் கூறப்பட்டுள்ளது.

“விண்ணவர்சூழ் பெருந்தொழு விளங்கிவரும் ஓதாளன்

வண்ணமணி மார்பன் மகராசன் சென்னிமலை

புதல்விமுத்து வேலம்மாள் பூதலத்தில் மாமயிலாள்

விதமான கொடுமணல் வேந்தன் கந்தசாமி

இன்பமுள்ள மனைவியென்று எல்லோருந் தானுரைக்க

அன்புப சாரமுடன் அழுதளிக்கும் பூமகள்தான்" (கந்தசாமிக்காதல், கண்ணி - 305)

பெருந்தொழுவூர் ஓதாளன் குலத்துச் சென்னிமலை என்பானின் மகள் முத்துவேலம்மா என்னும் மங்கை நல்லாள் கொடுமணலை ஆளும் மன்னன் கந்தசாமியின் மனைவி எனக் குறிப்பிடப் பெறுகின்றாள். இது ஏனைய காதல் நூலுக்கு இல்லாத புதுமையாகும்.

தலைவன் வேட்டைக்குச் செல்லும் முன்பு தன் சுற்றத்தார் அனைவரையும் அழைத்து விருந்து கொடுக்கிறான். இவ்விருந்தினை அனைவருக்கும் பரிமாறுபவள் தலைவனின் மனைவி என்று இக்காதல் இலக்கியத்தில் சுட்டப்பட்டுள்ளது.

\section{முடிவுரை}

தமிழ் இலக்கியங்களில் அகம் சார்ந்த பாடல்களே மிகுதி. தொல்காப்பியர் சுட்டிய அகமரபுகளான களவு, கற்பு என்னும் நிலைகளைப் பிற்காலத்தில் தோன்றிய அகப்பொருள் இலக்கண நூல்கள் விரித்து பேசுகின்றன. சங்க இலக்கியங்களில் உள்ள அகப்பொருள் மற்றும் புறப்பொருளையும் கருவாகக் கொண்டு காதல் சிற்றிலக்கியங்கள் தோற்றம் பெற்றன. காதல் என்ற சொல்லிற்கு அன்பு, காமவிச்சை முதலான பொருள்களைத் தருகிறது சென்னைப் பல்கலைக்கழகப் பேரகராதி. சங்க இலக்கியத்தில் மானிடக் காதலாகவும், பக்தி இலக்கியத்தில் இறைக்காதலாகவும், சிற்றிலக்கியக் காலத்தில் மீண்டும் மானிடக் காதலாகவும் காதல் பாடுபொருள் மாற்றம் பெற்றது. கண்ணியமைப்பில் பாடப்பட்ட இக்காதல் 


இலக்கியங்கள் சிற்றிலக்கியங்கள் தொண்ணூற்றாறு என்னும் பட்டியலில் இடம்
பெறவில்லையாயினும், சுட்டி ஒருவர் பெயர்கொளப் பெறார் என்ற அகமரபை மீறி வேட்டைவினையையும் இணைத்துக் காதலையும் வீர்தையும் ஒருசேரக் காட்டுகின்றன. மனுநீதிக்காதல், கந்தர் காதல், கதிர்காமக் காதல் போன்றவை பாடுபொருள் அமைப்பில் வேறுபட்டுள்ளன. இருப்பினும், தமிழர் வாழ்வியலில் இரு கண்களாகப் போற்றப்பட்ட காதலையும் வீரத்தையும் இணைத்த இலக்கியமாகக் காதல் சிற்றிலக்கியம் விளங்குகிறது.

\section{References}

Kodumanal Literatures, (1981), S. Rasu Pathipagam, Department of Archeology, Government of Tamil Nadu, India. Ponnusamy, M., (2004), Short literature history, Indhu Pathipagam, Chennai, India.

Subramaniyan, S.V., (1984), Tamil literary genre and format, Tamil Pathipagam, Chennai, India.

Tholkapiyam - Porulathigaram, (1969), Ilampuranar urai, South India Saiva Siddhanta Works Publishing Society, Chennai, India.

\section{Funding}

No funding was received for conducting this study.

Conflict of interest

The Author has no conflicts of interest to declare that they are relevant to the content of this article.

\section{About The License}

(C) The author 2020. The text of this article is open access and licensed under a Creative Commons Attribution 4.0 International License

\section{Cite this Article}

R. Veerapathiran, Insights on Romantic Medieval Literature, Indian Journal of Multilingual Research and Development, Vol 1, Iss 1 (2020) 27-33. DOI: https://doi.org/10.34256/ijmrd2014 